\title{
ESTUDO DAS NEUROPATIAS PERIFÉRICAS NO ESTADO DE SÃO PAULO DE 1939 A 1985
}

\author{
AMILTON ANTUNES BARREIRA *, WLSON MARQUES JR. **
}

\begin{abstract}
RESUMO - Constam do presente trabalho estudos sobre neuropatias periféricas de pesquisadores radicados no Estado de São Paulo, publicados a partir das décadas de trinta e quarenta. Maior impulso das investigaçōes na área ocorreu com o surgimento do periódico Arquivos de Neuro-Psiquiatria. Oswaldo Freitas Juliāo pode ser considerado o autor que iniciou os referidos estudos e suas contribuiçōes mais importantes se fizeram em relaçăo à hanseníase e à doença de Andrade, embora tenha também publicado trabalhos sobre outros tipos de neuropatias periféricas. Horacio Martins Canelas contribuiu sobremaneira para o estudo de diferentes neuropatias, em particular das por deficiência de vitamina B12. Uma série de trabalhos sobre o tema, publicada por neurologistas radicados no Estado, é sumariada. Apresentamos ainda, um catảlogo dos principais centros universitários em que há grupos de neurologistas que se dedicam preferencialmente ao estudo das doenças neuromusculares em São Paulo e uma bibliografia selecionada sobre neuropatias, de investigadores do referido Estado.
\end{abstract}

PALAVRAS-CHAVE: história da neurologia, história das neuropatias periféricas, neuropatias periféricas em Sāo Paulo.

Studies on peripheral neuropathies in the State of São Paulo, Brazil, from 1939 to 1985

ABSTRACT - Studies on peripheral neuropathies by investigators residing in the State of Såo Paulo, Brazil, and published since the 1930 and 1940 decades until 1985 were revised in the present survey. Investigations in the area were greatly encouraged by the appearance of the joumal Arquivos de Neuro-Psiquiatria(São Paulo). Oswaldo Freitas Julião may be considered the author who began these studies in the State and his most important contributions were related to leprosy and to Andrade disease, although he also published papers on other types of peripheral neuropathies. Horacio Martins Canelas also made a very important contribution to the study of different neuropathies, especially those due to vitamin B12 deficiency. A series of papers on neuropathies published by neurologists residing in the State is summarized. We also present a catalogue of the major university centers where groups of neurologists preferentially devote their time to the study of neuromuscular disease in São Paulo and a selected bibliography about neuropathies by investigators from this State.

KEY WORDS: history of neurology, history of peripheral neuropathies, peripheral neuropathies in São Paulo.

O estudo das neuropatias periféricas no Estado de São Paulo remonta à década de trinta. A criação da Cadeira de Neurologia desdobrada da de Psiquiatria e Moléstias Nervosas na Faculdade de Medicina de São Paulo em 1935, sob a regência de Enjolras Vampré pôde abrir a perspectiva do nascimento de uma Escola de Neurologia no Estado. Dando sequência ao trabalho de Vampré e contribuindo com suas qualidades pessoais ao desenvolvimento da neurologia em São Paulo, os Professores Adherbal Tolosa e Paulino Watt Longo assumiram a direção, após concurso público, respectivamente, da cátedra e do Serviço de Neurologia da Faculdade de Medicina da Universidade de São Paulo e da cátedra de Neurologia da Escola Paulista de Medicina.

A partir dos anos quarentas a regularidade das publicações sobre neuropatias periféricas passou a ser assegurada com o surgimento - em parte até como efeito do surgimento - e publicação contínua do periódico Arquivos

Departamento de Neurologia, Psiquiatria e Psicologia Médica da Faculdade de Medicina de Ribeirão Preto da Universidade de São Paulo (USP): *Professor Associado; **Professor Assistente. Aceite: 22-outubro-1996. Dr. Amilton Antunes Barreira - Faculdade de Medicina de Ribeirão Preto - 14049-900 Ribeirão Preto SP Brasil. 
de Neuro-Psiquiatria (São Paulo). Tal periódico começou a ser publicado, e se manteve, graças aos ingentes esforços e à direçāo do Professor Oswaldo Lange, então Livre-Docente.

Ao final da década de trinta, Oswaldo Freitas Julião inicia suas publicaçōes sobre a lepra nervosa ${ }^{55,58-60,63}$. A tese de livre-docência de Juliāo, Contribuição para o estudo do diagnóstico clínico da lepra nervosa (1945), é um marco no estudo das neuropatias periféricas no Estado de São Paulo. Um dos autores desta revisão (AAB) ouviu de Sérgio Pereira Novis ( Congresso Brasileiro de Neurologia, Rio de Janeiro, 1974) que o Professor Raymond Garcin afirmava que "aprendera a palpar nervos periféricos com Julião". A produção científica de Julião incluiu o estudo de aspectos neurológicos de diferentes neuropatias. Interessantemente se deteve na análise das neuropatias hipertróficas, cujo diagnóstico dependia, na época, somente de semiologia neurológica acurada. Estudou também a neurite hipertrófica de Déjérine-Sottas ${ }^{7}$ a amiloidose portuguesa ${ }^{11}$, que passou a diagnosticar após a vinda de Corino de Andrade ao Brasil. Wilson Brotto ${ }^{61}$ chama a atenção em relação a dois casos estudados por Julião e Antonio Couceiro, em 1940, ao destacar a importância do diagnóstico diferencial da lepra nervosa com a amiloidose portuguesa, escrevendo que aqueles dois casos se apresentavam com "perturbaç̃̃es motoras, amiotrofias, arreflexias, distúrbios da sensibilidade superficial, graves alteraçōes ao exame elétrico nos nervos e músculos, distúrbios esfinctéricos e sexuais e alteraçōes discretas do líquor cefalorraqueano, nos quais a suspeita de lepra nervosa pôde ser afastada". Julião escreveu ainda a propósito da síndrome de Landry nos anos quarentas ${ }^{7,66}$, precedendo as publicaçð̄es de José Lamartine de Assis e Antonio Spina França Netto em $1953^{3.9}$. Deixou ainda trabalhos sobre indiferença congênita generalizada à dor ${ }^{13}$ - hoje classificada no grupo das neuropatias sensitivas e autonômicas hereditárias - e porfiria aguda intermitente ${ }^{79}$. A par do seu interesse pelas neuropatias periféricas, Julião era neurologista diferenciado, didata reconhecido e foi Professor Adjunto de Neurologia na Faculdade de Medicina da Universidade de São Paulo, Titular na de Sorocaba da Pontifícia Universidade Católica de São Paulo e na de Ciências Médicas da Universidade Estadual de Campinas. Juliāo pode ser considerado o iniciador do estudo das neuropatias periféricas no Estado de Såo Paulo.

A importância de Horacio Martins Canelas, Professor Titular aposentado da Faculdade de Medicina da Universidade de São Paulo, na neurologia do Estado e do País é indiscutível. As suas contribuições se fizeram presentes no estudo de grande número dé afecçôes neurológicas, aí incluídas as neuropatias periféricas. A utilizaçāo do palestesiômetro, para quantificar a sensibilidade vibratória, foi pioneira em nosso meio mas, ainda hoje, infelizmente, os métodos psicofísicos de avaliação e quantificação da sensibilidade não têm aqui tido cultores. Canelas se deteve a estudar as características semiológicas e resposta à terapêutica das neuropatias carenciais e da mielopatia associada à deficiência de vitamina $B_{12}$. Tais estudos se estenderam desde a década de quarenta, alcançando os anos sessentas ${ }^{4,6,122029}$. Publicou estudos pioneiros sobre as neuropatias diabéticas, utilizando em sua investigąão biópsias de nervo em preparações incluídas em parafina ${ }^{74.75}$. Em colaboração com Spina-França entendeu que o aumento da proteinorraquia total na neuropatia diabética não é devido a alterações peculiares de uma ou outra fração protéica do líquido cefalorraqueano (LCR), nem está na dependência de modificaçōes do proteinograma no soro e não pode ser explicado em função direta da intensidade do quadro neurológico ${ }^{23}$. Apresentou contribuiçð̄es a respeito da amiloidose portuguesa ${ }^{4}$, um tipo de neuropatia sensitiva ${ }^{36}$ e sobre a terapêutica da neuropatia arsenical através do uso da penicilamina ${ }^{42}$. Certamente o interesse de Canelas pelo estudo das neuropatias carenciais foi um dos fatores motivadores para Milberto Scaff, atual Professor Titular da Faculdade de Medicina da Universidade de São Paulo, desenvolver suas teses de doutorado e livre-docência a respeito da mielose funicular.

Revisão sobre "polineurites" e polirradiculoneurites foi publicada por Spina-França em 1951, relacionando extensa gama de etiologias. Na mesma revisão encontram-se referências a importantes trabalhos clássicos sobre a síndrome de Guillain-Barrécs.

Além de Juliāo, outros neurologistas e investigadores de São Paulo estudaram a neuropatia da hanseníase. Ainda em 1937, Nelson de Souza Campos e Longo ${ }^{57}$ estudaram as atrofias circunscritas à eminência tenar como manifestação inicial e residual da lepra. O referido estudo é documentado através de detalhado exame elétrico dos músculos inervados pelos nervos radial, mediano e ulnar. Concluíram a favor do envolvimento preferencial do nervo ulnar nas referidas atrofias. Em 1950, Brotto documenta casos de reversão de dores secundárias ao comprometimento do nervo ulnar e do ciático em hansenianos, através da injeção de sulfato de amônia associado a procainamida ${ }^{61}$. A convite, duas revisōes importantes sobre o comprometimento nervoso da lepra foram realizadas ${ }^{62.03}$. Uma por Brotto, em 1954, apresentada durante a sessão comemorativa do $20^{\circ}$ aniverśrio da Sociedade Paulista de Leprologia e outra por Juliāo e Abrahão Rotberg, em relatório à Primeira Reunião Internacional de Neurologia Tropical, realizada em Buenos Aires em 1961 e publicada em 1963. Em 1962 Spina-França e Brotto ${ }^{21}$ publicaram estudo identificando: aumento do teor de albumina no LCR de pacientes com formas inespecíficas de hanseníase, com maior frequência nos casos de curta evolução e aumento de beta-globulina em acometidos pela forma tuberculoide. Em 1962 e1963 Brotto e Spina-França ${ }^{2124}$ evidenciaram aumento do teor de gama-globulina 
no LCR de hansenianos lepromatosos, considerado secundário ao aumento que ocorre no sangue de tais pacientes. Na metade da década de sessenta Brotto publicou trabalho no qual catalogou as alternativas oferecidas pelo eletrodiagnóstico na lepra ${ }^{\text {(4) }}$. No início da década de setenta, Ceme Ferreira Jordy e Walter Belda ${ }^{\text {t5 }}$ estudam a velocidade de condução nervosa de 16 hansenianos. Surpreendem-se com o pequeno número de casos que apresentavam redução daquela velocidade e com outros com aumento e outros, ainda, com valores normais.

As neuropatias inflamatórias, além dos já citados trabalho de Julião, tiveram o início do seu estudo no Estado de São Paulo através de contribuiçðes de Assis e Assis e Spina-França (atual Professor Titular aposentado da Faculdade de Medicina da Universidade de São Paulo), no ano de 1953, com a proposta do uso de ACTH e cortisona para tratamento da síndrome de Guillain-Barre $\$$,9. José Antonio Levy e Wilson Luiz Sanvito (atual Professor Pleno da Faculdade de Ciências Médicas da Santa Casa de São Paulo), descreveram 3 casos de síndrome de GuillainBarre, em 1965, chamando a atençāo para os seus aspectos clínicos e evolutivos ${ }^{67}$. $O$ envolvimento bulbar foi registrado em 1959 por Paulo Pinto Pupo e colaboradores, incluindo José Geraldo de Camargo Lima, atual Professor Titular aposentado da Escola de Medicina da Universidade Federal Paulista ${ }^{27}$. Referências à síndrome de MillerFisher foram feitas por Paulo Eurípedes Marchiori e colaboradores, incluídos entre os quais Scaffe Assis e por Luis Antonio de Lima Resende e colaboradores, inclứdo Takassu Sato, já na década de oitenta ${ }^{\text {47,51 }}$. Os últimos, então ligados à área de neurologia da Faculdade de Medicina de Ribeirão Preto. Registro de 2 casos de polirradiculoneurite recidivante foi efetivado na primeira metade da mesma década pelo grupo liderado por Assis ${ }^{48}$. Spina-França e Sívio Saraiva estudaram o proteinograma do LCR e do soro de pacientes com síndrome de Guillain-Barré e encontraram aumento de alfa-globulinas. Segundo os autores, as modificaçðes que observaram no teor das diversas fraçōes proteicas do LCR poderiam ser secundárias a alteraçőes verificadas no soro e decorrer de distúrbios do mecanismo de ultrafiltraçåo das proteínas do sangue para o LCR, por modificaçðes da barreira hêmato-liquớrica ${ }^{18}$. Merecem referência os trabalhos do grupo liderado por Spina-França sobre positividade de reaçðes para sífilis no LCR em paciente com polirradiculoneurite $(1963)^{25}$ e na meningomielorradiculopatia esquistossomótica (1971) ${ }^{37} \mathrm{e}$ a associação registrada por Carlos A. M. Guerreiro, da Faculdade de Ciências Médicas da Universidade Estadual de Campinas, da polirradiculoneurite e malária $(1985)^{54}$.

No início da década de quarenta, Assis e Paulo Cunha Cintra ${ }^{72}$ publicaram trabalho sobre a síndrome de Ramsay-Hunt e, no início da de cinquenta, Roberto Melaragno Filho publicou revisão sobre diagnóstico e tratamento das paralisias faciais periféricas ${ }^{56}$. A contribuição da Escola Paulista de Medicina para o estudo de distúrbios do nervo facial tem sido de grande importância. Em 1954, Longo e Jorge Armbrust de Lima Figueiredo (atual Professor Titular aposentado da Faculdade de Medicina de Ribeirão Preto) propuseram o boqueio do gânglio estrelado como alternativa terapêutica para tratamento das paralisias faciais periféricas rebeldes ${ }^{10}$. Posteriormente o grupo liderado por Camargo-Lima estudou os primeiros casos de síndrome de MelkersonRosenthal registrados no País e constatou relação entre otite média tuberculosa e paralisia facial periférica ${ }^{39}$. Mais recentemente, através da orientação científica de João Antonio Maciel Nóbrega, Jovany Luis Alves de Medeiros padronizou método para avaliação eletroneuromiografica do nervo facial do ponto de vista da normalidade e demonstrou a sua utilidade em situaçōes patológicas.

Uma curiosa e, aparentemente até então única, associação de neuralgia dos nervos trigêmeo, glossofaringoe vago, foi documentada por Rolando Ângelo Francisco Tenuto, João Teixeira Pinto e Roger Perret Gentil em $1955^{14}$. O tratamento com a secção dos dois últimos nervos e cavólise resultou em reversão da sintomatologia.

Em 1984, João Aris Kouyoumdjian registrou a associação entre mononucleose infecciosa e amiotrofia neurálgica ${ }^{52}$.

O estudo das consequências da porfiria aguda intermitente sobre o sistema nervoso periférico tem tido contribuições importantes da parte da Universidade de São Paulo, em particular do Departamento de Neurologia e Neurocirurgia da Faculdade de Medicina. Na segunda metade da década de cinquenta, Assis chamava a atenção para a importância do método de Watson e Schwartz para o diagnóstico ${ }^{13}$. Na década seguinte, Assis, Juliāo e Eurico Thomaz de Carvalho ${ }^{79}$ publicaram trabalho descrevendo os aspectos clínicos e evolutivos de 6 pacientes acometidos por porfiria aguda intermitente, com estudo necroscópico de 3 deles. Abrão Anghinah e Jamil Sallum ${ }^{80}$, na mesma década, publicaram estudo necroscópico de um caso. Significativa contribuição foi efetivada pelo grupo do Instituto de Química da Universidade de São Paulo, liderado por Etelvino Bechara, em associação com Marchiori, evidenciando a importância de radicais livres no desencadeamento das lesões na porfiria e definindo que determinados medicamentos, capazes de aumentar a produçāo daquele tipo de radicais, podem agravar o quadro clínico do paciente com neuropatia porfírínica.

Oestudo das neuropatias sensitivo-motoras hereditárias fez parte das preocupações dos neurologistas radicados em São Paulo desde os idos de quarenta. No primeiro volume dos Arquivos de Neuropsiquiatria, em 1943, Longo, Paulo Pinto Pupo e Dante Giorgi apresentam o estudo de três casos de irmãos acometidos por amiotrofia 
do tipo Charcot-Marie, dois dos quais associados a sinais piramidais ${ }^{1}$. No mesmo volume, Henrique San Mindlin e Melaragno Filho discutem a nosologia das heredodegenerações espinocerebelares, registrando dois casos de ataxia do tipo Pierre-Marie. Tanto em um como no outro trabalho, os autores defendem a teoria unicista, de parentesco entre as doenças familiares da medula e do neurônio periférico e entre as citadas heredodegeneracões. Fortemente influenciados pelas idéias de Marinesco, reforçadas no Brasil pelo pensamento de Austregésilo, que admitia o referido parentesco entre aquelas doenças - tendo como entidade central a ataxia de Friedreich -, aqueles autores se contrapunham ao pensar de Barraquer Ferré e Purves-Stewart, dentre outros eminentes neurologistas da época. Juliăo, em 1952, reunindo 3 casos que observou na França, do que é hoje denominado de neuropatia sensitivo-motora hereditária do tipo I (doença de Charcot-Marie-Tooth do tipo I), faz extensa revisão sobre o assunto, incluindo duas biópsias do ramo sensitivo do nervo radial efetuadas naquele Pais, verificando a existência de neuropatia hipertrofica ${ }^{7}$. Levy, em 1962, publicou o estudo de 4 casos de "moléstia" de Charcot-Marie, com a demonstração de achados sugestivos de miopatia primária ou distrofia muscular progressiva à biópsia de músculo, associados a evidências de atrofia neurogênica. Aproximando os seus achados aos de Haase e Shy, sugere ser aquela "moléstia" uma neuromiopatia ${ }^{19}$. No início dos anos setentas Charles Peter Tilbery e colaboradores, incluindo José Luiz Alonso Nieto, registram a associação, na mesma família, entre "doenças de Charcot-Marie e de Friedreich"38.

Em 1955 Julião e Brotto estudam o caso de um paciente acometido por indiferença congênita generalizada à dor que, provavelmente, correspondia à hoje denominada neuropatia sensitiva e autonômica hereditária do tipo II $^{13}$. No mesmo ano Armbrust-Figueiredo e Juvenal Silva Marques estudam um segundo caso, adotando a denominação de indiferença congênita universal à dor ${ }^{\text {s2 }}$. Sanvito, em 1968, fez o primeiro registro de casos de neuropatia sensitiva e autonômica do tipo I em nosso meio ${ }^{32}$. Scaff, Ferri e Canelas publicam, em 1970, estudo eletroforético e imunoeletroforético do soro sanguíneo na "neuropatia radicular sensitiva hereditária"36.

Ao final da década de trinta Adherbal Tolosa e Carlos Virgilio Savoy publicam trabalho sobre polineurite sulfamidica, adotando a terminologia, então universal, para as hoje denominadas polineuropatias ${ }^{81}$. Anghinah, Francisco B. de Jorge, Francisco Forti e Antonio F. Ferrari, em 1973, confirmam que a quantidade de arsênico no nervo periférico normal, se existente, $\epsilon$ indosável, utilizado o método de Kingsley-Chaffert ${ }^{4}$. No ano seguinte, associados a Canelas e sem a participação de Ferrari, estudam caso de polineuropatia arsenical, cuja resposta à terapêutica com penicilamina aconteceu em associação com a progressiva queda dos níveis de arsênico, inclusive em amostras de nervo periférico nas quais se fez a dosagem do referido metal ${ }^{42}$.

As neuropatias associadas às vasculites foram estudadas por Clemente B. Pereira, Hélio Lôbo e Sanvito, ao registrarem 3 casos de poliarterite nodosa com envolvimento do sistema nervoso periférico ${ }^{34}$.

Um apreciável conjunto de trabalhos experimentais sobre respostas normais e patológicas dos nervos periféricos foi publicado por Eros Abrantes Erhart a partir dos anos cinquentas $5.27,30,31,33,35,45,49.7$. Na década de oitenta, acompanhado por Ciro Ferreira da Silva e Cláudio Fava Chagas, se dedicou a estudos experimentais, agora documentados através de microscopia eletrônica. Nos dias de hoje elegantes estudos experimentais sobre a regeneraçăo de nervos periféricos são realizados na Universidade de São Paulo sob o comando de Ciro Ferreira da Silva. A utilização de fatores de crescimento, ativos em relação a estruturas componentes das fibras nervosas, tem trazido resultados positivos quanto d̀ referida regeneração.

Spina-França realizou estudo descritivo e morfométrico do $7^{\circ}$ comparado ao do $5^{\circ}$ gânglio cervical , respectivamente de Hydrochoerus capybara e Cavia cobaya. Concluiu que o volume médio dos neurônios sensitivos do gânglio espinhal da capivara era maior que o da cobaia na proporçảo de 8,20:1 em contraposição ao número de neurônios do gânglio cervical da primeira, maior na proporção de 3,34:1 em relação ao da segunda. Considerando que a relação da superfície corporal das capivaras para as das cobaias estudadas era de $27,4: 1$, documentou uma desproporf̧ão entre o número de neurônios no gânglio da capivara c a respectiva superfícic corporal. Entendeu que tal desproporção era justificada pelo maior volume dos neurônios sensitivos da capivara, o que levaria a uma compensação quando considerado o número de neurônios contidos nos gânglios das duas espécies. No mesmo estudo, Spina-França comparou os neurônios da coluna cinzenta anterior da medula espinal e os piramidais da zona central do córtex, encontrando diferenças menos acentuadas nas dimensões que as observadas em relação aos neurônios sensitivos das duas espécies. Os dendritos da capivara, por sua vez, tinham as características de serem mais numerosos, mais longos, mais ramificados e mais espessos que os da cobaia ${ }^{83}$.

A realização do exame elétrico do nervo e músculo se iniciou em nosso meio ainda na década de trinta. Paulino Longo realizou detalhado estudo sobre o envolvimento de músculos inervados pelos nervos radial, mediano e ulnar, em pacientes acometidos por hansenfase, concluindo pelo envolvimento do nervo ulnar. Savoy e Maria Elisa Bierrenbach Khoury ${ }^{2}$, já no primeiro número dos Arquivos de Neuropsiquiatria, apresentam trabalho sobre a "reação fibrilar" nas afeçōes da coluna cinzenta anterior da medula. Savoy e Brotto marcaram sua passagem pela neurologia de São Paulo sobretudo na condição de especialistas na realização do exame elétrico. 
A eletroneuromiografia (ENMG) começou a ser realizada na década de sessenta na Faculdade de Medicina da Universidade de São Paulo por Jairo de Andrada e Silva. Outro pioneiro da área foi Eduardo Carlos de Figueiredo Ferraz. Abrão Anghinah atuou na ánea. Edgard Silva Lusvarghi trabalhou vários anos no Hospital das Clínicas da Faculdade de Medicina da Universidade de São Paulo, tendo sido responsável pela área de ENMG. Lusvarghi teve morte prematura. Chefia aquele setor nos dias de hoje Mário Wilson lervolino Brotto. Maria Teresa Alves Hirata, que teve treinamento com Brotto, trabalha no setor.

Ceme Jordy, na década de sessenta, iniciou as atividades na área de ENMG no Hospital Såo Paulo, Escola de Medicina da Universidade Federal da São Paulo. Em 1976, João Antonio Maciel Nóbrega passou a exercer as atividades de eletroneuromiografista naquele hospital. Inicialmente utilizou o seu próprio equiparnento até que, através de doação, o hospital pôde ter o seu. No momento, além de Nóbrega, trabalha naquele hospital Gilberto Manzano.

No início dos anos sessentas, José Luiz Alonso Nieto, que trabalhou com Julião em Sorocaba, iniciou as suas atividades de neurologista e eletroneuromiografista no Hospital do Instituto de Assistência Médica dos Servidores Públicos Estaduais. Assumiu a chefia do setor até o início dos anos noventas, quando se aposentou. Assumiu, então o setor, por concurso público, a eletroneuromiografista Roseli Aparecida Freire, que fez seu treinamento no Hospital das Clínicas da Faculdade de Medicina de Ribeirão Preto sob a orientação de Ronaldo Francisco Herrera e Wilson Marques Júnior e no Hospital São Paulo com Nóbrega e Manzano.

A partir do iń́cio da década de 90, Berenice Cataldo Valério, apos especialização com Nóbrega, no Hospital Sāo Paulo, ativou o setor de ENMG da Santa Casa de Sāo Paulo.

Nélson de Bortoli, treinado por Alonso-Nieto, iniciou as atividades de ENMG no Hospital das Clínicas da Faculdade de Medicina de Ribeirāo Preto no final dos anos setentas. Em meados da década de oitenta, assumiu o setor, Hernera, neurologista com residência médica no Hospital das Clínicas da Faculdade de Medicina da Universidade de Săo Paulo e treinado em ENMG por Lusvarghi. Herrera se reciclou com Carlos Roberto de Faria em Goiânia e com Asa Wilboum na "Cleveland Clinic", em Cleveland. Em 1992 assumiu a chefia, Marques Júnior, cuja formaçāo incluiu um ano de treinamento em ENMG sob orientação do próprio Herrera, e outro de aperfei̧̧oamento com Austin Sumner em New Orleans. No momento, além de Marques-Júnior, trabalha no setor, Adriana Leoni Couto Rosa Dutra de Oliveira, que fez um ano de treinamento em ENMG em Campinas, sob a orientação de Anamarli Nucci e outro em Ribeirāo Preto, sob a do próprio Marques-Júnior.

Nucci é responsável pelo setor de ENMG e de doenças neuromusculares da Faculdade de Ciências Médicas da Universidade de Campinas.

Na Faculdade de Medicina de Botucatu, o setor de ENMG é chefiado por Resende, que fez seu treinamento com Roberto Sica, em Buenos Aires.

Kouyoumidjian, da Faculdade de Medicina de São José do Rio Preto, teve treinamento em ENMG no Hospital Sara Kubitshek em Brasilia.

A formaçāo de áreas de trabalho nos hospitais acadêmicos, dirigidas especificamente para o estudo das neuropatias periféricas, se iniciou na segunda metade da década de setenta, quando Camargo-Lima passou a setorizar a Clínica Neurológica da então Escola Paulista de Medicina. Alberto Alan Gabbai se responsabilizou pelo setor de doenças neuromusculares, integrando seu trabalho com o de Nóbrega e, em anos posteriores, com o de Beni Schmidt (patologia muscular) e de Acari Bulle de Oliveira. Acoplada com um forte empenho em relação ao curso de p6sgraduação, a setorização tem rendido frutos com produção científica regular. Nóbrega tem formado continuamente especialistas em eletroneuromiografia. Sob sua orientação, especialistas na área têm podido elaborar suas monografias de mestrado e teses de doutorado, utilizando ou padronizando métodos eletroneuromiográficos com regularidade e continuidade únicas no País. As atividades científicas de Gabbai e seus orientados se dirigiram mais para as doenças musculares. A produção de monografias e teses tem se feito com regularidade.

No Hospital das Clínicas de São Paulo, Marchiori criou, em 1983, ambulatório para acompanhamento de pacientes acometidos por polirradiculonueurites e, há cerca de 4 anos, Hirata passou a ser responsável pelo ambulatório de neuropatias periféricas, supervisionando também o atendimento dos pacientes de enfermaria. Ana Maria Tsanaclis passou a se responsabilizar pela interpretação das biópsias de nervo. Os resultados de tal trabalho têm sido publicados e apresentados em eventos científicos.

No Hospital do Instituto de Assistência Médica dos Servidores Estaduais, Joăo Roberto Pereiro deu impulso às atividades da área de doenças neuromusculares na década de setenta. O empenho investigativo, no referido hospital, se faz mais em direção às doenças musculares.

No Hospital das Clínicas da Faculdade de Medicina de Ribeirão Preto, Barreira iniciou as atividades da área de doenças neuromusculares na primeira metade da década de oitenta. Entre 1983 e 1985 foram criadas 4 disciplinas de pós-graduação, as primeiras do País, dedicadas ao estudo das neuropatias periféricas. Em 1985 , 
foi criado o ambulatório de doenças neuromusculares e, pouco tempo depois, determinou-se que 6 leitos da enfermaria de neurologia seriam utilizados para investigação, diagnóstico e tratamento das doenças neuromusculares. A integraçāo com o setor de ENMG se fez desde o início. Ainda em 1983, biópsias de nervo com cortes semi-finos começaram a ser realizadas e, há 7 anos, foi criado o Laboratório de Neurologia Aplicada e Experimental. Em junho de 1992 integrou-se ao grupo Leila Chimelli, neuropatologista que, a convite do Departamento de Patologia, transferiu-se da Faculdade de Medicina da Universidade Federal Fluminense para a Faculdade de Medicina de Ribeirâo Preto-USP. Nos últimos 11 anos foram apresentadas publicamente 7 monografias de mestrado, 5 teses de doutorado e uma tese de livre-docência, a respeito de neuropatias periféricas. O grupo realizou, em Ribeirāo Preto, três simpósios sobre temas específicos em neuropatias periféricas. Um em 1989, sobre neuropatias diabéticas, com a participaçāo de Peter James Dyck, o segundo em 1991 sobre neuropatias inflamat́rias, com as de Austin J. Sumner e Franz van der Meché e o terceiro em $1995 \mathrm{com}$ a de Gérard Said. Foi fundada, durante o primeiro simpósio, a Sociedade Brasileira para Estudo do Nervo Periférico, da qual Barreira $\epsilon$, no momento, presidente.

No Hospital das Clínicas da Faculdade de Ciências Médicas da Universidade Estadual de Campinas, Nucci é responsável pelo setor de doenças neuromusculares e o empenho investigativo tem se feito, preferencialmente, em direçăo ao estudo das miopatias. Há a participaçāo do neuropatologista Luciano Queiróz, que interpreta as biópsias de nervo e músculo.

No Hospital das Clínicas da Faculdade de Medicina de Botucatu da Universidade Estadual Paulista, Resende coordena a área de doenças neuromusculares, havendo perspectivas promissoras no campo investigativo. do Rio Preto.

Kouyoumdjian é responsável pelo setor de doenças neuromusculares da Faculdade de Medicina de São José

Nos próximos anos a produção científica na área de neuropatias periféricas no Estado de São Paulo deverá aumentar dos pontos de vista qualitativo e quantitativo e em relação a trabalhos originais. Os centros de excelência, de aplicação e pesquisa, deverão se diferenciar mais. Novos centros dedicados a área deverăo surgir e outros se desenvolverem no Estado. As informaçōes recolhidas, para a redaçāo do presente capítulo, constituem indícios favoráveis a esta interpretação.

Ao finalizar o presente trabalho, não temos dúvidas de que cometemos algumas injustiças - involuntárias, naturalmente. Há insuficiências de informaçōes e talvez tenhamos cometido enganos. Năo seguimos, propositalmente, as normas técnicas para referências bibliográficas com a finalidade de dar ênfase à cronologia das publicaçōes. Publicą̧ões de neurologistas de São Paulo em periódicos ou publicações de outros Estados e no exterior, sobre neuropatias, nem sempre foram referidas. Intencionalmente nāo destacamos trabalhos que predominantemente tenham enfocado o sistema nervoso autônomo. Estudos eventuais sobre neuropatias periféricas, efetivados por estudiosos não ligados às neurociências, foram omitidos. O espaço das neuropatias focais foi bastante reduzido, embora se reconheça o importante papel profissional de determinados neurocirurgiōes que se dedicarn a tal área.

Aceitaremos, agradecidos, qualquer sugestão pertinente para futuras inodificaçōes do texto, informaçōes adicionais e propostas de correçðes de desvios que, porventura, tenharnos cometido.

A colaboraça de Edymar Jardim, atual Professor Titular e Chefe do Departamento de Neurologia, Psiquiatria e Psicologia Médica da Faculdade de Medicina de Ribeirão Preto, foi inestimável: além de preciosas informações, ofereceu-nos a sua biblioteca para consultas. A colaboração de Spina-França e Camargo-Lima foi de extrema importância para a elaboraçăo do presente artigo. Nơbrega, Marchiori, Alonso-Nieto e Assis também contribuíram com informą̧ðes que foram utilizadas no texto. A todos o nosso sincero agradecimento.

\section{BIBLIOGRAFIA SELECIONADA DE PUBLICACð̃̃ES DE NEUROLOGISTAS DO ESTADO DE SÃO PAULO SOBRE NEUROPATIAS PERIFÉRICAS}

A presente bibliografia reúne trabalhos publicados no periódico Arquivos de Neuropsiquiatria (São Paulo) de 1943 a 1985 e outros trabalhos de neurologistas radicados em São Paulo.

\section{Arquivos de Neuro-Psiquiatria (Sāo Paulo)}

1943

1. Paulino Watt Longo, Paulo Pinto Pupo \& Dante Giorgi. Possível parentesco entre as moléstias familiares com lesōes predominantes na medula e moléstias familiares com lesōes no neurônio periférico.1(1):43-52.

2. Carlos Virgilio Savoy \& Maria Elisa Bierrenbach Khoury. Particularidades e valor do exame elétrico nas afeçóes do corno anterior da medula. Reaçăo fibrilar.1(2):119-132. 
1948

4. Michel Abu Jamra, Horacio Martins Canelas \& J. M. Taques Bittencourt O ácido fólico no tratamento da síndrome nervosa da anemia perniciosa. Estudo do comportamento da sensibilidade vibratória. 6(4):301-333.

1950

5. Eros Abrantes Erhart. Nerve compression as an essencial factor in causing ischaemic and post-ischaemic paraesthesiac. 8(1):3942

1952

6. Horacio Martins Canelas. Distuirbios neurológicos nos estados carenciais aquílicos. 10(1):1-40.

7. Oswaldo Freitas Juliāo. Étude sur la névrite hypertrophique progressive de Déjerine-Sottas. 10(2):221-246.

1953

8. José Lamartine de Assis. Sindrome de Guillain-Barré. A propósito de três casos, sendo um tratado pelo ACTH. 11(1)133144.

9. José Lamartine de Assis \& Antonio Spina França Netto. Síndrome de Guillain-Barré. A propósito de 5 casos tratados com ACTH e cortisona. 11(4):414-419.

1954

10. Paulino Watt Longo \& Jorge Armbrust Figueiredo. O bloqueio do gânglio estrelado nas paralisias faciais periféricas rebeldes a outras terapêuticas. 12(4):441-442.

1955

11. Oswaldo Freitas Juliảo \& Constantino Mignone. Amiloidose primária com comprometimento meningo-radiculoneurítico. 13(1):1-12.

12. Horacio Martins Canelas \& José Antonio Levy. Tratamento das neuropatias desmielinizantes aquílicas pelo ACTH. Resultados imediatos e tardios. 13(4):296-302.

13. Oswaldo Freitas Julião \& Wilson Brotto. Indiferença congênita, generalizada à dor. 13(4):338-342.

14. Rolando A. Tenuto, João Teixeira Pinto \& Roger Perret Gentil. Neuralgia dos nervos trigêmeo, glossofaringeo e vago. 13(4):343-346.

1957

15. José Lamartine de Assis. Porfiria aguda intermitente. Valor do teste de Watson e Schwartz para o diagnóstico. 15(1):35-45. 1959

16. José Lamartine de Assis \& Gilles Henry Duchêne. Complicaçōes neurológicas da vacinaçăo anti-rábica. 17(3):235-250.

17. Paulo Pinto Pupo, José Geraldo de Camargo Lima, Eliova Zukerman, Ceme Jordy, Nelson Alvares Cruz \& Fernando Menezes Braga. Formas bulbares da síndrome de Guillain-Barré. 17(4):364-376.

1961

18. A. Spina-França e Sylvio Saraiva. Alteraçōes do proteinograma do líquido cefalorraquidjano na polirradiculoneurite. 19(4):287-294.

1962

19. José Antonio Levy. Moléstia de Charcot-Marie-Tooth: conceito clínico-patológico atual. 20(3):131-136.

20. Spina-França A \& Canelas HM. Proteinograma do líquido céfalorraqueano na mielose funicular. 20(3):183-199.

21. Spina-França A \& Brotto W. Proteinograma no líquido cefalorraqueano na lepra. 20(4):279-288.

22. Eros Abrantes Erhart. Normal nerve-fibers in the distal segment of nerves completely separated from the proximal stump for more than six months. 20(4):289-306.

1963

23 A. Spina-França \& Horacio M. Canelas. Proteinograma do líquido cefalorraqueano na neuropatia diabética. 21(2):88-86.

24 Brotto W \& Spina-França A. Proteinograma do líquido cefalorraqueano na lepra lepromatosa. 21(3):172-180.

25. Maria Immina Valente \& A. Spina-França. Positividade de reaçōes para sífilis no líquido cefalorraqueano em um caso de polirradiculoneurite. 21(3):203-208.

1964

26. Abrăo Anghinah \& Alberto Alencar Carvalho. Cinesioterapia nas polineuropatias periféricas em crianças. 22(1):61-65. 1965

27. Eros Abrantes Erhart \& Cecil José Rezze. Nerve fibers in isolated segments of dog ulnar nerves after complete brachial plexotomy and periaxilar artery sympathectomy. 23(2):82-90.

28. Eros Abrantes Erhart \& Cecil José Rezze. The neuroma-like structure of the long time severed and isolated nerve stumps. 23(2):91-94.

29. Horacio Martins Canelas, Nelson de Carvalho \& Arnaldo Gama da Rocha. Vitamin $B_{12}$ in the pathogenesis of subacute combined degeneration of the spinal cord. 23(4):253-260.

1966

30. Eros Abrantes Erhart \& Cecil José Rezze. Effect of experimental devascularization on peripheral nerves. 24(1):7-11.

31. Eros Abrantes Erhart \& Cecil José Rezze. Further discussion on the "next-growing" nerve fibers which repopulate the distal segment of nerves completely separated from the proximal for more than six months. 24(1):91-96. 
32. Wilson Sanvito. Acropatia úlcero-mutilante fanilial, consideraçōes a propósito de três casos. 26(1):39-47.

33. Eros Abrantes Erhart, Cecil José Rezze \& Walter Biazzoto. The ectopic newly-formed nerve fibres which repopulate the longtime denervared and atrophic chick skeletal muscle. 26(3):187-194.

1969

34. Clemente A. B. Pereira, Hélio Lóbo \& Wilson Luiz Sanvito. Manifestaçð̃es neurológicas periféricas da periarterite nodosa. Relato de três casos. 27(3):227-232.

1970.

35. Eros Abrantes Erhart, Antonio Carlos Berardi, José Furlani Fausto Bérzin \& Walter Biazotto. Nerve conduction patterns determined electronically for analysing peripheral nerves behavior "in vivo". 28(2):118-124.

36. Milberto Scaff, Rubens G Ferri \& Horacio Martins Canelas. Hereditary sensory radicular neuropathy: an electrophoretic and immunoelectrophoretic study of the blood serum. 28(4):362-366.

1971

37. Milberto Scaff, Danieli Riva \& Antonio Spina-França. Meningomielorradiculopatia esquistossomótica. 29(2):227-233.

1972

38. Charles Peter Tilbery, Dócio Cassiani Altimari, Luiz Carlos Gagliardi-Ferreira \& José Luiz Alonso Nieto. Associação na mesma famflia das doenças de Charcot-Marie-Tooth e de Friedreich. 30(2):158-165.

1973

39. Octavio Lemmi, Josê Geraldo Camargo Lima, Milton Medeiros \& Pedro Camilo de A. Pimentel. Síndrome de MelkerssonRosenthal. Relato de 4 casos e revisão da literatura. 31(1):38-42.

40. Eliova Zukerman \& Sérgio Zatz. Esclerose lateral amiotrófica e neurossífilis. 31(3):214-219.

41. Abrăo Anghinah, Francisco B. de Jorge, Francisco Forti e Antonio F Ferrari. Arsênico em nervos periféricos normais do bomem. 31(3):193-199.

1974

42. Abrảo Anghinah, Horacio Martins Canelas, Francisco B. de Jorge \& Francisco Forti. Polineuropatia periférica arsenical: tratamento compenicilamina 32(3):240-245.

43. Josk Geraldo Camargo Lima \& N. Alvares Cruz. Paralisia facial periférica e otite média tuberculosa. 32(3):253-256.

1975

44. Eduardo M. Azevedo, Milberto Scaff, Horacio Martins Canelas \& Antonio Spina-Franq̧a. Type I primary neuropathic amyloidosis (Andrade, Portuguese).A clinical and laboratory study of 21 cases. 33(2):105-118.

45. Eros Abrantes Erhart, Marcus Castro Ferreira, Antonio Tedesco Marchese \& Pedro Puech Leāo. Microsurgical nerve sutures improve rehabilitation and may prevent Wallerian degeneration. 33:267-293

1977

46. Aguinaldo Gonçalves \& Jeova Barros Silva. Irmandade afetada pela atrofia muscular peroneal de Charcot-Marie-Tooth com possível variante do fenómeno de antecipaçăo. 35(2):167-171.

1982

47. Paulo Euripedes Marchiori, Milberto Scaff, Dagoberto Callegaro, D Figueira \& José Lamartine de Assis. Síndrome de Miller Fisher: relato de um caso. 40(3):268-271.

1983

48. Paulo Euripedes Marchiori, Dagoberto Callegaro, Milberto Scaff, Antonio Zambon \& José Lamartine de Assis. Polirradiculoneurite recidivante: relato de 2 casos. 41(2):208-211.

49. Eros Abrantes Erhart, Ciro Fenreira da Silva \& Cláudio Fava Chagas. Proper microsurgical nerve suture may impede wallerian degeneration of completely transected nerves. An electron microscopic study. 41(3):215-227.

50. José Luiz Alonso, Açucena Atalla, Maria J. Cavaliere, Sônia Maria Gagioti \& Maria A. A. Lorenti. Polineuropatia por parathion. Estudo clfrico, eletrofisiológico e histológico de um caso. 41(3):292-308.

1984

51. Luis Antonio de Lima Resende, Reinaldo JR Battistuta, Takassu Sato \& Nelson de Bortoli. Síndrome de Miller Fisher: relato de um caso. 42(2):171-174.

52. Jožo Aris Kouyoumdjian. Neuropatia do plexo braquial (neuralgia amiotrófica) e mononucleose infecciosa - registro de um caso. 42(3):289-293.

1985

53. Sergio Rosemberg, F. Arita \& C. Campos. Distrofia neuroaxonal infantil: diagnóstico em vida por biópsia conjuntival. 43(1): 48-54.

54. Carlos A. M. Guerreiro, Nubor O. Facure, Fernando L.Gonçalves Jr., Luis Jacintho da Silva, Marcelo Carvalho Ramos \& Rogério de Jesus Pedro. Polirradiculoneurite e malária. Relato de um caso. 43(2):214-216. 


\section{Medicina Clinica (Barcelona)}

55. Oswaldo Freitas Julião. Les elements du diagnostic de la lèpre aerveuse. 15:349,1952.

Pinheiros Terapêutica

56. Roberto Melaragno Filho. Diagnóstico e princípios do tratamento das paralisias faciais periféricas. 5:1-10, 1953.

Revista Brasileira de Leprologia

57. Nelson de Souza Campos \& Paulino W. Longo. Atrophia circunscripta aos músculos da cminência thenar como marifestaçåo inicial e residual da lepra. $5(1): 29-51,1937$.

58. Oswaldo Freitas Julīo. Sobre um caso de lepra nervosa. 78(1):77-120,1939.

59. Oswaldo Freitas Juliảo \& Carlos Virgilio Savoy. Eletrodiagnóstico na lepra. 10(3):273-302, 1942.

60. Oswaldo Freitas Juliāo. Mielodisplasia e lepra nervosa. 11(4):319-342, 1943.

61. Wilson Brotto. Tratamento das neuralgias cubitais e ciáticas em hansenianos pelo sulfato de amônio associado à procaina 18(2):84-93,1950.

62. Wilson Brotto. Aspectos neurológicos da lepra. 22(2):135-144,1954.

63. Oswaido Freitas Julião \& Abrahăo Rotberg. O comprometimento neurológico na lepra. 31 ( 1 e 2):5-33,1963.

64. Wilson Brotto. Eletrodiagnóstico na lepra. 33(1 a 4):23-34,1965.

65. Ceme Ferseira Jordy \& Walter Belda. Consideraçð̄es sobre a conduçăo nervosa periférica na hanseníase. 37(1 a 4):61-70,1970.

\section{Revista Clínica de São Paulo}

66. Oswaldo Freitas Julião. A propósito da sínđrome de Landry. 16:189-185,1944.

\section{Revista do Hospital das Clínicas da Faculdade de Medicina de São Paulo}

67. Jose Antonio Levy \& Wilson Luiz Sanvito. Polirradiculoneurite: considerações sobre 3 casos. 20:239-244, 1965.

\section{Revista de Medicina}

68. A. Spina França Netto. Polineurites e polirradiculoncurites. Diagnóstico e tratamento. 35 (200):236-248, 1951.

\section{Revista Médica de Såo Paulo}

69. Luis Marques-Assis. Dor nas afeçð̋es neurológicas perif́́ricas. 51:27-31,1967.

\section{Revista de Neurologia e Psiquiatria de São Paulo}

70. Venturino Venturi. Consideraçōes sobre uma diplegia facial de provável causa escarlatinosa.5:1-16,1939.

71. Oswaldo Freitas Julião \& Antonio Couceiro. Estudo de dois casos de meningorradiculite espinhal crônica. 6:141-156,1940.

72. José Lamartine de Assis \& Paulo Cunha Cintra. Síndrome de Ramsay-Hunt. 8:179 - 182, 1942.

\section{Revista Paulista de Medicina}

73. José Lamartine de Assis. Afecçðes do sistema nervoso periférico e capacidade profissional. 45(4):397-406, 1954.

74. Horacio Martins Canclas, Eduardo Carlos Figueiredo Ferraz, Dotina Reichhardt Epps, \& Josto Teixeira Pinto. Neuropatia diabetica: consideraçóes sobre 33 casos. 49(3):169-187,1956.

75. Horacio Martins Canelas. Neuropatia điabética. 62(1):29-42,1963.

76. Horacio Martins Canelas. Quantificąāo clínica da sintomatologia neurológica 63(3):178-184,1963.

77. Eros Abrantes Erhart Novos conceitos sobre degeneraçäo de fibras nervosas periféricas. 63(3):209,1963.

78. Oswaldo Freitas Juliäo. Aspectos clínicos da neưropatia amiloide.63(5):349-352,1963.

79. José Lamartine de Assis, Oswaldo Freitas Juliāo \& Eurico Thomas de Carvalho. Porfiria aguda intermitente: estudo de seis casos. 65(1):41-56,1964.

80. Abrāo Anghinah \& Jamil Sallum. orfiria aguda intermitente: estudo anátomo-patológico de um caso. 72(2):78-84, 1968.

\section{São Paulo Médico}

81. Adherbal Tolosa \& Carlos Virgilio Savoy. Sobre um caso de polineurite sulfamídica 12:269 - $279,1939$.

\section{Senra Médica}

82. Jorge Armbrust Figueiredo \& Juvenal da Silva Marques. Indiferença congênita à dor. 10:231-238, 1955.

\section{Zeitschrift für Zellforschung}

83. Antonio Spina França Netto. Dimensioni e numero dei neuroni in relazione alla mole somatica. Confronti tra due roditori di mole somatica molto differente. 36:222-234,1951. 